\title{
Recherche Qualitative en Santé et Modèles \\ Infectieux : Cas d'une étude Anthropologique de la Co-infection TB/VIH dans les CAT (Centre Anti-Tuberculeux) d'Adjamé et de Bouaké en Côte d'Ivoire
}

\section{Gnamien Konan Bah Modeste,}

Docteur en Anthropologie Biologique, Assistant à l'Unité de Formation et de Recherche en Sciences Sociales et Humaines, Université Jean Lorougnon Guede de Daloa, Côte d'Ivoire

\section{Kouame Atta,}

Maitre de Conférences en Anthropologie Biologique, Institut des Sciences Anthropologiques de Développement, Université Félix Houphouët Boigny de Cocody-Abidjan, Côte d'Ivoire

\section{Yoro Blé Marcel,}

Professeur Titulaire en socio-anthropologie à l'Institut des Sciences Anthropologiques de Développement, Université Félix Houphouët Boigny de Cocody-Abidjan

Doi:10.19044/esj.2019.v15n24p417 URL:http://dx.doi.org/10.19044/esj.2019.v15n24p417

\section{Résumé}

Cet article porte sur les voies méthodologiques empruntées dans le cadre d'une recherche qualitative portant sur une affection à dimension bipolaire, en l'occurrence la co-infection TB/VIH. Il ambitionne montrer une fois de plus la flexibilité et la valence méthodologique élevée de la démarche qualitative. Par essence le VIH-Sida se caractérise par des affections opportunistes caractéristiques de l'immunodépression, on est alors au stade de sida. De ce fait la lecture de cette affection ne devrait se contenter d'une posture uniquement unipolaire comme généralement constaté. Jusqu'à présent les orientations qualitatives ont ciblé l'homogénéité du VIH-Sida au détriment de son hétérogénéité qui est pourtant évidente. L'exposé indique comment la trajectoire méthodologique allant de la triangulation, tout en mobilisant des paramètres rétrospectifs et prospectifs en lien au déroulement synchronisé ou diachronique des évènements infectieux, et aux fins d'entrevues semi-dirigées, a permis d'atteindre l'objectif envisagé. Ainsi, cet exercice entend partager son expérience de recherche anthropologique au sujet de la co-infection 
TB/VIH, pour qu'elle soit soumise à critique de manière à contribuer de façon efficiente à l'élan de capitalisation des recherches qualitatives.

Mots-clés : Recherche qualitative, Tuberculose, VIH, Modèles infectieux

\section{Qualitative Health Research and Infectious Models: Case of an Anthropological Study of TB / HIV Co-infection in the Adjamé and Bouaké Anti- Tuberculosis Centers (CAT) in Côte d'Ivoire}

\section{Gnamien Konan Bah Modeste,}

Docteur en Anthropologie Biologique, Assistant à l'Unité de Formation et de Recherche en Sciences Sociales et Humaines, Université Jean Lorougnon Guede de Daloa, Côte d'Ivoire

Kouame Atta,

Maitre de Conférences en Anthropologie Biologique, Institut des Sciences Anthropologiques de Développement, Université Félix Houphouët Boigny de Cocody-Abidjan, Côte d'Ivoire

\section{Yoro Blé Marcel,}

Professeur Titulaire en socio-anthropologie à l'Institut des Sciences Anthropologiques de Développement, Université Félix Houphouët Boigny de Cocody-Abidjan

\section{Abstract}

This paper focuses on the methodological pathways used in qualitative research on a bipolar disorder in the case of TB / HIV co-infection. This is one of the goals of the premium and heat method. HIV-AIDS is characterized by opportunistic affections of immunodepression at the stage of AIDS. An understanding of this affection should not be seen from one perspective. Until now, the qualitative orientations have targeted the homogeneity of HIV-AIDS to the detriment of its heterogeneity, which is nevertheless obvious. The presentation shows how the methodological trajectory from triangulation, while mobilizing retrospective and prospective parameters in relation to synchronized or diachronic unfolding of infectious events, and for semidirected interviews, made it possible to achieve the objective of this study. This paper, however, aims to provide anthropological research on TB / HIV 
co-infection which is subject to criticism so as to contribute efficiently to the capitalization momentum of qualitative research

Keywords: Qualitative research, Tuberculosis, HIV, Infectious models

\section{Introduction}

La méthode qualitative est utilisée lorsque l'on vise à comprendre ou à décrire un phénomène qui est particulièrement peu connu ou exploré (Morse \& Field, 1995). Elle permet de produire et d'analyser des données descriptives comme les paroles écrites ou orales, ainsi que les comportements observables des personnes étudiées (Taylor \& Bogdan, 1984, p.5). La principale caractéristique de cette approche est de privilégier l'avis des acteurs sociaux dans l'appréhension des réalités sociales (Mayer \& Deslauriers, 2000). Et ce, dans tous les champs disciplinaires y compris le domaine de la santé. Par « recherches qualitatives en santé », l'on entend tout type de recherche, menée dans le champ de la santé, mettant en œuvre des modes de collecte de données et des méthodologies d'analyses, pouvant être qualifiées de qualitatives, au sens où elles permettent d'accéder à la compréhension, en profondeur, du phénomène investigué que les approches quantitatives sont incapables de déceler (Kivits et al., 2016 ; Kouamé, 2017). Fort du succès de la recherche qualitative en santé, on constate sa sollicitation constante allant souvent à sa systématisation en complément à la recherche quantitative et surtout dans la perspective positiviste où le social « régénère » le biologique. Elle a donc pu modéliser la question sanitaire en élément qualitativement documenté et saisir toutes les attitudes et pratiques qui gravitent autour de tous les cursus infectieux. Cette recherche qualitative a su se positionner dans l'actualité sanitaire en se rendant incontournable dans la recherche sur le VIH-Sida et a contribué à sa stabilité sociale.

Dans cet élan d'explications des processus infectieux, elle s'est vue confrontée à différents modèles ou configurations, d'abord unipolaires (infection unique) et aussi bipolaires (co-infection). Dans le cas de cette étude, il a été jugé opportun de se demander : comment la recherche qualitative a-telle procédé pour interroger les affections bipolaires et assurer la traçabilité, la dissociation et l'individualisation des logiques culturelles mobilisées dans un environnement doublement référencé ? Il s'agit ici en clair, de présenter l'itinéraire méthodologique emprunté ou peaufiné pour traiter les maladies faisant intervenir deux agents infectieux. Cet exercice est envisagé à travers une étude anthropologique sur la co-infection TB/VIH réalisé en Côte d'Ivoire dans les CAT de Bouaké et d'Adjamé. Les variables qui ont été documentées sont les perceptions, les états affectifs et rupture biographique, l'environnement social du patient et la dimension thérapeutique.

Les raisons qui soutiennent la tenue de cette étude sont multiples ; 
- Au plan personnel, cette étude tire sa source des comportements observés dans une famille ayant hébergé un proche atteint de coinfection TB/VIH et de notre souci de comprendre ces comportements.

- Au niveau scientifique, elle permet de confronter la démarche qualitative à l'étude d'une affection bipolaire afin de mettre en lumière les avantages et de déceler les éventuelles pesanteurs. Cette étude offre à cet égard des éléments d'analyse qui illustrent les enjeux méthodologiques et théoriques de l'approche de cette double infection.

- Les zones choisies pour l'étude, à savoir les régions d'Abidjan et de Bouaké, hébergent plus du tiers de la population et concentrent environ la moitié des cas de tuberculose et plus de $50 \%$ des cas de co-infection TB/VIH en Côte d'Ivoire.

Après cette phase introductive, il revient dès à présent, selon le fil conducteur de cet article, à exposer la justification de l'usage de la démarche qualitative, la construction de l'objet, les théories mobilisées, le choix des enquêtés, l'opération d'analyse, les résultats et la conclusion.

\section{Justifivation de L'approche qualitive}

La caractéristique essentielle des méthodes qualitatives porte sur leur ouverture et leur souplesse. Ainsi la raison fondamentale qui justifie leur usage dans cette étude est la grande flexibilité pour adopter l'approche nécessaire pour découvrir l'inattendu ou explorer en profondeur les thèmes particuliers. Elles sont ainsi à même d'expliquer ce qui est susceptible d'éclairer la causalité d'un phénomène qui se répète; les raisons de son apparition dans tel contexte plutôt que dans tel autre. «En permettant aux gens d'exprimer librement leurs opinions, points de vue et expérience, les méthodes qualitatives visent à cerner la réalité telle que la définit le groupe à étudier, sans imposer à la population un questionnaire ou un cadre pré structuré » (Maier \& Goergen, 1994, p.3). L'on définit généralement la recherche qualitative en sciences sociales comme " une activité permettant d'acquérir des connaissances sur les réalités culturelles et sociales définies, dans des contextes précis »(Baudry, 1975, p.50). Selon Denzin et Lincoln (2005), la recherche qualitative permet au chercheur d'étudier les objets dans leur milieu naturel, d'interpréter les phénomènes ou d'en tirer le sens à partir des explications que leur donne la population concernée. Dans le cadre de ce projet d'article, il a été donc pertinent d'adopter une approche qualitative en s'introduisant dans l'environnement des co-infectés TB/VIH-sida et des professionnels de la santé, afin d'identifier, compartimenter et classer leurs pratiques et leurs opinions. Cela a permis de capter et adresser toutes les intentions et logiques 
d'actions culturellement polarisées qui pourraient nous éclairer sur cette problématique. Ces considérations justifient le choix que nous avons fait, d'utiliser les techniques qualitatives.

\section{Théories mobilisées}

Il s'agit de l'approche par la théorie de l'engagement et de la distanciation et la théorie de la représentation sociale. L'usage ici de la théorie de l'engagement et de la distanciation se réfère à Elias (1993) qui la relie à une ambition de proposer une autre grille de lecture que celle de "l'application" qui a souvent servi à clôturer le débat en ne posant, à travers la défense ou la critique de l'anthropologie appliquée, que la question des conditions de possibilité d'un transfert de connaissances dans le registre de l'action. Cette position est jugée confortable et capable de contribuer à une nouvelle lecture et une version bipolaire du VIH-Sida par opposition à sa conception traditionnelle unipolaire. Elle s'est faite en trois étapes toujours inspirée d'Elias (1993).

- La première position réalise la conjonction d'une proximité à la fois par rapport à l'action et par rapport à l'analyse : on la qualifiera d'appliquée. Elle se met au service de la lutte contre le sida et adhère aux principes d'analyse de la médecine. La justification qu'elle se donne est l'amélioration de la santé publique par la connaissance de représentations et de pratiques autour du sida et des affections connexes et de ce qui lui est indiqué comme relevant de conduites à risque.

- La seconde position en est la symétrique dans la mesure où elle prend ses distances à la fois en termes d'action et d'analyse : on l'appellera critique. Elle n'est pas indifférente pour autant à la lutte contre le sida dans une version unipolaire, mais, d'une part, elle met en question les bases interprétatives sur lesquelles se fonde cette dernière au regard de l'émergence de sa forme bipolaire.

- La troisième position se présente comme l'association d'une proximité de l'action et d'une distance dans l'analyse : on la désignera comme impliquée. Elle s'efforce d'intervenir directement dans la prise en charge des problèmes liés à la maladie, tant au niveau des soins que de la prévention, sans pour autant adhérer aux présupposés des malades, des professionnels et des responsables visà-vis desquels sont adoptées des attitudes plus ou moins distanciées.

Il faut entendre par représentations sociales (Jodelet, 1989), les idées plus ou moins arrêtées que les individus se sont faites de la santé et de la maladie et qui concourent à faire un choix thérapeutique. L'importance de cette approche se justifie par le fait que les représentations sociales préparent à l'action et génèrent aussi un ensemble d'attentes normatives. Ainsi, elles 
permettent de comprendre et d'expliquer les liens sociaux et les pratiques sociales (Yoro, 2012).

\section{La construction de l'objet}

Tout en ne privilégiant pas les données numériques elles ont été d'un apport considérable dans la construction de notre objet de recherche. En effet, les données quantitatives couplées aux discours recueillis lors de la préenquête, dans le cadre d'une opération de triangulation, se sont avérées indispensables pour capter le chercheur en posture de prospection thématique. La triangulation est entendue au sens classique du mot, il s'agit d'obtenir des données différentes mais complémentaires sur un même sujet afin de mieux comprendre le problème de recherche (Morse, 1991). Denzin (1978) a mis en évidence trois types de triangulation : l'utilisation de sources de données différentes pour le temps, l'espace et les personnes ; l'utilisation de chercheurs différents ; l'utilisation de méthodes différentes en même temps. Il est donc possible de distinguer entre triangulation méthodologique, triangulation des sources d'information et triangulation des participants (Berger, Crescentini, Galeandro \& Crohas, 2010). La triangulation méthodologique utilisée ici est évoquée par Erzberger et Kelle (2003) qui affirment que les résultats de recherches qualitatives peuvent être vérifiés, ou corroborés, par des méthodes statistiques. En effet, le rapprochement de données épidémiologiques qui indiquaient les taux de co-infection à $20 \%$ et $22 \%$ respectivement dans les CAT d'Adjamé et de Bouaké (PNLT, 2015) aux données qualitatives qui relèvent la coexistence fréquente du virus du VIH et de l'agent de la tuberculose a suscité l'attrait sur cette pathologie et permis de révéler un modèle infectieux bipolaire à documenter. Se refusant à toute mixité et dans le souci de garantir l'identité qualitative, l'usage des données numériques s'est arrêté à ce niveau de démarrage de l'étude. Nous avons pu formaliser quelque peu les idées conçues et arrêter la question de départ autour des dispositions ou états affectifs orientant positivement ou négativement le déroulement de la vie quotidienne qui sont mobilisés dans la trajectoire du malade, au cours de la prise en charge de la co-infection TB/VIH dans les CAT d'Adjamé et de Bouaké. Notre trajectoire méthodologique a ensuite mené des escales au niveau du recensement des données existantes sur la question, la définition des objectifs généraux et la formulation de la problématique globale.

\section{La sélection des enquêtés et la conduite des entretiens / outils d'enquête}

Les paramètres qui ont été mobilisés pour la sélection des enquêtés sont d'ordre rétrospectif et prospectif. En effet, compte tenu du caractère curable de la tuberculose, nous avons ciblé des malades séropositifs avec un passé tuberculeux (rétrospective) et des malades présentant encore la coinfection TB/VIH-Sida (prospective). Cette approche permet de documenter 
l'histoire naturelle de la maladie, c'est-à-dire ses agents, son terrain, son cursus, mais aussi son histoire sociale, en d'autres mots, le contexte anthropologique et sociologique de son apparition, de sa transmission, de sa perception et de ce qui va devenir. Ce profil permet également de saisir les éléments des variables étudiées durant le passage de la dimension unipolaire à la dimension bipolaire et vice versa, le vécu de la dimension bipolaire et la possibilité de soustraire graduellement et dissocier les aspects anthropologiques spécifiques de chaque affection à la mesure de l'évolution infectieuse.

Le VIH/sida, couplé à la tuberculose dans notre cas, en Afrique se vit différemment qu'en Europe à cause des difficultés thérapeutiques et des représentations et autres clichés négatifs dont il fait objet (Kouamé, 2012). Il reste donc ici un sujet sensible et délicat, difficile à aborder, surtout quand il s'agit d'en parler avec des personnes directement concernées (Kouamé, 2010). La méthode non-probabiliste, non aléatoire et la technique d'échantillonnage de volontaires a été utilisée. Les sujets recrutés sont ceux qui ont accepté volontairement de participer à la recherche. Il a été vérifié que la demande de participation soit claire et accessible au plus grand nombre. Le corpus sur lequel le travail a été fait était constitué de 77 individus (dont 27 de sexe féminin) répartis en catégories de 7 infirmiers, 3 assistants sociaux, 6 médecins, 38 malades et 23 proches des patients.

Aussi, tel que précisé par Patton (1990), il n'existe pas de règles spécifiques pour la taille de l'échantillon de l'étude. Selon le même auteur, le nombre varie selon l'objet et la question de recherche, son enjeu, et selon ce qui peut être utile, crédible et fait avec le temps et les moyens disponibles. Sans rejeter l'aspect quantitatif, la recherche qualitative ne lui accorde pas la première place et donne plus d'importance à l'analyse des phénomènes sociaux ainsi qu'au sens que les personnes et les groupes leur attribuent (Deslauriers, 1991). Denzin et Lincoln (1994) vont dans le même sens en soulignant qu'en recherche qualitative, c'est plus la quantité et la profondeur des informations collectées qui importent que le nombre de participants. La taille de l'échantillon a été jugée suffisante quand l'effet de saturation est atteint, c'està-dire lorsque la poursuite de la collecte de données n'apporte plus de nouvelles informations au chercheur (Deslauriers, 1991 ; Morse, 1994).

L'outil de collecte utilisé est le guide d'entretien semi-directif dans le souci de révéler les expériences actuelles, passées et comprendre comment elles affectent le quotidien des malades. Ainsi, les entrevues semi-dirigées s'avèrent très pertinentes pour répondre à ces exigences. En effet, comme le disent Savoie-Zajc (1997) et Patton (2002), on est justifié de choisir l'entrevue de type qualitatif lorsque les données à recueillir sont des perceptions, des représentations et des évaluations personnelles. 
Son élaboration a gravi autour des symptômes dissociés de chaque affection avant d'aborder la dimension conjointe et sommative.

Il s'est agi pour la tuberculose :

- La toux,

- Les sueurs nocturnes,

- La perte de poids,

- La fièvre,

- Les émissions salivaires purulentes, blanchâtres et sanguinolentes.

Pour le VIH :

- La diarrhée,

- Le vomissement,

- Le manque d'appétit,

- L'amaigrissement,

- L'altération cutanée.

Ensuite plusieurs combinaisons des symptômes ci-dessus ont été faites pour aborder la dimension sommative en lien à la bipolarité de l'affection. Et ce, relativement aux perceptions, aux états affectifs et rupture biographique, à l'environnement social du patient et la dimension thérapeutique. En somme, nous avons dressé l'historique du profil épidémiologique de chaque patient, interrogé distinctement chaque affection à la mesure de son apparition et synchronisé les différents signes.

\section{Difficultés rencontrées}

Il n'est jamais aisé d'enquêter sur cette catégorie de malades où tous les détails sont importants. D'abord, la principale difficulté a été la lourdeur administrative dans un sens où l'accord du responsable de la structure est obligatoire, mais aussi celui des personnes interrogées. Ensuite, il a fallu rassurer les malades au sujet de la confidentialité de leur identité. Nous avons été aidés par les ONG qui exercent dans les deux CAT qui ont facilité notre immersion dans l'environnement des patients. En termes de leçons apprises, il est apparu que le bon déroulement de l'entretien portant sur une affection faisant intervenir deux agents infectieux dépend du travail effectué au préalable sur le guide d'entretien. Il est nécessaire d'identifier clairement au préalable, les questions pertinentes par rapport au sujet.

Une des difficultés ressenties au cours de l'entretien est de savoir se positionner face aux personnes interrogées, mais également de ne pas perdre le fil du guide d'entretien tout au long de la conversation. Il faut savoir rebondir sur les réponses données par « l'interviewé », éviter les amalgames entres les affections et pouvoir se recentrer sur les questions établies au départ. 


\section{Méthode d'analyse}

Deux méthodes d'analyse ont été retenues dans cet article, à savoir : la méthode compréhensive et l'interactionnisme.

Selon Emile Durkheim (1997), « la méthode compréhensive est une logique orientée vers la saisie du sens d'une activité ou d'un comportement ». Notre démarche compréhensive s'est appuyée sur la conviction de Weber (1995) que les sciences sociales (que Weber nomme «sciences de la culture ») diffèrent des sciences de la nature, en ce que l'homme est un être de conscience qui agit en fonction de sa compréhension du monde et des intentions qu'il a. Cette méthode nous a été utile dans la compréhension des comportements de santé des populations, des malades face à la co-infection TB/VIH-SIDA.

Selon David Le Breton (1985) : «Pour l'interactionnisme, l'individu est un acteur interagissant avec les éléments sociaux et non un agent passif subissant de plein fouet les structures sociales à cause de son habitus ou de la 'force' du système ou de sa culture d'appartenance » (p.46). Son utilisation dans le cadre de cette étude se justifie dans le sens où l'objet de l'étude, à savoir : la co-infection TB/VIH, fait interagir deux affections et le traitement implique l'interaction de plusieurs acteurs et plusieurs molécules.

\section{Traitement des données}

La conduite de l'opération de traitement des données s'est articulée autour de notre intention d'assurer la traçabilité de chaque variable à son agent infectieux et de saisir la dimension sommative. Les données extraites ont été traitées selon une démarche inductive inspirée de Mayer et Deslauriers (2000). Ensuite, une analyse du contenu et une analyse transversale ont été faites. L'analyse transversale a abouti à l'émergence de variables comme les perceptions, les états affectifs et rupture biographique, l'environnement social du patient et la dimension thérapeutique. La méthode d'analyse du contenu a pour objectif ultime de « déterminer la signification exacte du message étudié » (L'Écuyer, 1990, p.14) et toujours selon (L'Écuyer, 1987, p.50) de classer ou de codifier les données.

\section{Résultats Obtenus}

\section{Perceptions de la Co-infection TB/VIH chez les malades}

Rappelons que le corpus sur lequel nous avons travaillé était constitué de 77 individus (dont 27 de sexe féminin) répartis en catégories de 7 infirmiers, 3 assistants sociaux, 6 médecins, 38 malades et 23 proches des patients. Après avoir appliqué tout le processus méthodologique décrit plus haut, il est ressortit que tous les malades interrogés au CAT d'Adjamé comme au CAT de Bouaké décrivaient des symptômes relatifs à la première pathologie révélée, VIH ou tuberculose. Nous avons aussi remarqué, chez 
certains, que la corrélation était très vite établie entre la tuberculose et le VIH, faisant état d'une coopération, d'une complicité et d'une synergie prononcée entre les deux virus. Des verbatim, il appert que la co-infection TB/VIH est perçue comme une forme d'organisation infectieuse collective impliquant un lien d'intelligence entre le virus de la tuberculose et celui du Sida. Ces deux virus s'influencent mutuellement, agissent ensemble et créent un effet plus grand que la somme des effets attendus s'ils avaient opéré séparément ou indépendamment ou crée un effet que chacun d'entre eux n'aurait pas pu obtenir en agissant seul. Nombreux sont les séropositifs qui développent une tuberculose comme première expression du sida, dans la mesure où l'infection par le VIH constitue le plus fort facteur de risque de transformation d'une tuberculose latente en infection active, ou de rechute chez les patients ayant déjà subi un traitement. Par ailleurs, en cas de co-infection, chacune de ces maladies accélère le développement de l'autre, leur puissance destructrice cumulée étant supérieure à leur impact indépendant. De même que l'infection par le VIH favorise le passage rapide de la tuberculose infection à la tuberculose maladie, celle-ci accélère la progression de l'infection par le VIH (en augmentant la charge virale).

\section{Les états affectifs et rupture biographique}

Pour les malades, la tuberculose commence le plus souvent par les signes suivants : une fièvre, des maux de tête, une toux nocturne et persistante, des douleurs aux côtes et/ou à la poitrine, une diminution de la force, des crachats qui peuvent être purulents, blanchâtres, sanguinolents, collant sur la terre. Elle s'aggrave et le VIH se suspecte par la diarrhée ou le vomissement, le manque d'appétit et l'amaigrissement. Passer cette étape, le malade effectue une auto analyse de sa biographie et mobilise les dispositions ou états affectifs, orientant positivement ou négativement le déroulement de sa vie quotidienne. Selon les professionnels de santé du CAT de Bouaké et d'Adjamé, la plupart des malades au su de leur situation de co-infecté énoncent ces propos sommairement ancrés. L'accentuation du bouleversement de la personnalité du malade co-infecté est tributaire d'une mobilisation sommative des attributs de la tuberculose et du VIH. On note la saisine des éléments d'altération biographique à la fois du VIH et de la tuberculose intensifiant la rupture biographique traditionnellement vécue par les personnes atteintes de pathologies chroniques.

\section{Environnement social du patient}

La mobilisation mondiale autour du VIH-Sida a permis une certaine stabilisation et relative acceptation sociale. Mais sa cohabitation fréquente, constatée ces dernières années avec la tuberculose, tend à inverser cette tendance. Les récits illustrent les dures épreuves auxquelles sont soumis les 
malades co-infectés ; on constate que de nombreux problèmes liés étroitement à l'image véhiculée à propos de cette pathologie, en lien à sa dimension sommative, continuent de peiner le vécu des malades. La tuberculose devient par conséquent un indicateur populaire du VIH-Sida et le caractère insalubre qui lui est associé, en rapport avec la toux grasse et, compte tenu de sa volatilité, pousse l'entourage à placer le malade dans un isolement. L'image du malade est modelée par le caractère incurable du VIH associé à la pénibilité et à la volatilité de la tuberculose. Il s'ensuit une redéfinition constante de la notion de contagion qui alimente les comportements de marginalisation et d'évitement rapportés. Dans bien des cas, il est clair qu'à la souffrance physique se greffe une souffrance psychique allant jusqu'à l'idéation suicidaire (Kouamé, 2009).

\section{La dimension thérapeutique}

La dimension additive de la prise en charge thérapeutique est à la base de la pénibilité évoquée par les malades. L’omniprésence des tradithérapeutes dans le parcours de soin des malades est avérée et motivée dans bien des cas par la bipolarité de l'affection. Ceci montre bien que les représentations profanes de la maladie, ainsi que les critères de décisions amenant les sujets à consulter un praticien sont de principaux déterminants des choix de l'instance thérapeutique. (Yoro, 2012).

À cet égard, il apparaît nécessaire d'élargir l'analyse de l'usage conjointe des ARV (antirétroviraux) et antituberculeux aux facteurs d'ordre social et culturel, dimensions qui ne sont pas encore suffisamment prises en compte dans les

études scientifiques, à notre connaissance.

\section{Discussion des Résultats}

L'itinéraire méthodologique décrit dans cet article nous a permis de montrer que la confrontation à cette double peine (TB et VIH) dans la vie d'une personne co-infectée engendre des changements tellement importants que le pronostic vital est engagé. Outre les changements d'habitudes imposés par l'affaiblissement physique et les nécessités du traitement, pouvant entraîner des conséquences profondes sur le statut social, professionnel et familial du patient, ce qui peut être difficile à vivre, la personne malade se retrouve confrontée à l'éventualité de la reconsidération de sa personnalité entrainant des ruptures biographiques. Ces répercussions sont également exprimées par une étude menée en Afrique de l'ouest, mais axée sur l'aspect genre (Bonfoh et al., 2010). Selon cette étude, les personnes atteintes de la tuberculose ou du VIH/SIDA sont stigmatisées et contraintes à vivre quelque peu en périphérie sociale. Leurs statuts se transforment et se fragilisent, leur recours à des liens sociaux, aux systèmes de tradithérapie, à la spiritualité et 
au secteur médical informel semble être une issue de secours de la maladie. Poursuivant dans ce sens Vidal (1992), dans une étude entamée à Abidjan sur les représentations du Sida et les caractéristiques anthropologiques de la prise en charge des séropositifs, affirme que le discours sur la nature de la maladie contient par ailleurs, bien évidemment, des références à la tuberculose qui influencent parfois profondément les représentations de la contagion, de la guérison et, plus généralement du message préventif propre au sida.

Cet article a aussi révélé que l'environnement social des malades coinfectés est lourdement affecté. C'est dans ce sens qu'Adam et Herzlich (2001) écrivaient que «certaines maladies chroniques comme certains handicaps disqualifient les personnes qui en sont atteintes, celles-ci faisant l'objet d'une stigmatisation ». La stigmatisation des malades chroniques devient ainsi une « invariante culturelle » dans diverses communautés. Une étude sur les séropositifs à la Guadeloupe montre qu'ils ont la mort dans l'âme (Bombereau, 2005), ce qui engendre en eux une sorte de stoïcisme. Les malades co-infectés font face à une double crainte : l'image sociale négative des deux maladies et la lourdeur des traitements. Ils ont un problème d'intégration sociale. Cette dynamique de dévalorisation sociale des personnes séropositives a été montrée au Burkina Faso (Charmillot, 1997). La situation d'évitement créée par la stigmatisation entraîne des abandons de traitement (Desclaux, 2003), mais surtout des résistances aux traitements. Pour le malade chronique hébergeant sida et TB, la lassitude face au poids social et l'acharnement thérapeutique, engendre un isolement progressif du malade par rapport à son réseau social (Kouamé, 2010). Les résultats sont corroborés par une étude menée en contexte ivoirien sur la gestion de la maladie par Yéo et al (2012). L'objectif de cette étude était d'améliorer le pronostic des schizophrénies, et il ressort que la famille demeure une pièce incontournable dans la demande de soins et la prise en charge des malades schizophrènes. Poursuivant dans ce sens, Kouadio (2015) à travers une étude dénommée "Environnement psycho-social et maladies chroniques, cas de la tuberculose multi résistante » a obtenu des résultats similaires. Ses enquêtes ont révélé de profonds changements dans les dynamiques familiales, professionnelles et sociales des patients et le caractère fondamental de l'apport de l'environnement psychosocial (milieu social) dans le soutien psychologique recherché par les patients.

Enfin les différentes escales thérapeutiques constatées dans le présent article nous font penser à une étude menée en Côte d'Ivoire, en région Bété, par Yoro (2012). Cette étude démontre qu'au regard d'un périple thérapeutique, les itinéraires se caractérisent à la fois par une succession d'étapes et d'un cumul thérapeutique. 


\section{Conclusion}

Retenons que la recherche qualitative a été à la hauteur de l'analyse des questions liées à la co-infection TB/VIH dans les CAT d'Adjamé et de Bouaké. La triangulation méthodologique a été utilisée pour la construction de l'objet. Ensuite, la théorie de l'engagement et de la distanciation et la théorie de la représentation sociale ont été mobilisées. Les paramètres qui ont été mobilisés pour la sélection des enquêtés sont d'ordre rétrospectif, et prospectif. La méthode non-probabiliste, non aléatoire et la technique d'échantillonnage de volontaires ont été utilisées. Les sujets recrutés sont ceux qui ont accepté volontairement de participer à la recherche. L'élaboration du guide d'entretien a gravi autour des symptômes dissociés de chaque affection avant d'aborder la dimension conjointe et sommative. Deux méthodes d'analyse ont été retenues, à savoir : la méthode compréhensive et l'interactionnisme.

Il ressort des résultats de l'approche qualitative appliquée à la coinfection VIH/Tuberculose que les malades établissent une corrélation entre le VIH et la tuberculose en décrivant les symptômes des deux infections uniquement par ceux de la première infection révélée, faisant ainsi état d'une coopération et d'une synergie d'action entre les deux virus. Pour eux, réciproquement l'une des infections accélère l'action de l'autre et entraîne des rechutes chez les patients ayant déjà subi un traitement. En cas de co-infection, leur puissance destructrice cumulée est supérieure à leur impact indépendant. Les états affectifs et les ruptures biographiques des malades sont fonction des bouleversements subits en lien avec la nature bipolaire de l'affection. La coinfection VIH/Tuberculose et l'image négative des deux infections impactent l'environnement social des malades et accentuent les difficultés relationnelles avec l'entourage. La tuberculose place les malades dans des rapports d'évitement de l'entourage liés à la peur de la contagion et aggrave la stigmatisation et l'ostracisme déjà liés au VIH/Sida. Les choix thérapeutiques opérés sont fonction de la bipolarité et de la pénibilité de l'affection. De même le recours aux tradithérapeutes est motivé par ces deux réalités liées à l'affection.

\section{References:}

1. Adam, P. \& Herzlich, C., (dir.) (2001). Sociologie de la maladie et de la médecine. Paris : Nathan/HER.

2. Beaudry, L. (1975).Guide de recherche à l'intention des militants.Montréal: Centre coopératif de recherche en politique sociale. 
3. Berger, E., Crescentini, A., Galeandro, C., \& Crohas, M. (2010). Actes du congrès de l'Actualité de la recherche en éducation et en formation (AREF), Université de Genève, septembre.

4. Bombereau, G. (2005). Représentationssociales du VIH/sidaen Guadeloupe et recommandations à l'usage de la santé publique la peurou la mort dansl'âmedans les Antilles françaises. Thèse de doctorat, université Laval Québec et université René - Descartes-Paris 5, Paris, France.

5. Bonfoh, B., Ould,Taleb, M., Kéita, M., Durand-Bourjate, Y., Kablan, C., Betsi, A. N., Nguessan, S., Schwaerzler, P., \& Cisse, G. (2010). Représentation, mode de gestion et stratégies de luttecontre la Tuberculose\& le VIH/SIDA en Afrique de l'Ouest, [enligne]. http://www.csrs.ch/atelierlausanne/com/906a Bonfoh FR.pdf (page consultée le 13 Janvier 2014).

6. Charmillot, M. (1997). Les savoirs de la maladie. Education à la santé en contexte africain, Cahiers de la Section des sciences de l'éducation. Pratiques et théorie; 81 , Genève.

7. Denzin, N.K. \& Lincoln, Y.S. (1994). Handbook of qualitative research. California. Thousand Oaks, Sage Publications.

8. Denzin, N.K. (1978). The research act: A theoretical introduction to sociological methods.New York: McGraw-Hill.

9. Desclaux, A. (2003). Stigmatisation, discrimination : que peut-on attendre d'une approche culturelle ? Actes de la table ronde organisée le 29 novembre 2002 à l'Unesco-Paris « VIH/SIDA stigmatisation et discrimination : une approche anthropologique ». Etudes et rapports, série spéciale, ${ }^{\circ} 20$, Division des politiques culturelles et du dialogue interculturel.

10. Deslauriers, J.P. (1991). Recherche qualitative : guide pratique. Montréal: McGraw-Hill.

11. Elias, N. (1993). Engagement et distanciation. Paris, Fayard (1ère édition 1983).

12. Erzberger, C. \& Kelle, U. (2003). Making inferences in mixed methods : the rules of integration. In A. Tashakkori \& C. Teddlie (Eds.), Handbook of Mixed Methods in Social Behavioral Research (pp. 457488). Thousand Oaks, London, New Dehli : Sage Publications.

13. Jodelet, D. (1989). Les représentations sociales. Paris : Presses Universitaires de France.

14. Kivits, J., Frédéric B., Cécile F., \& Myriam W. (2016). Les recherches qualitatives en sante. Éditeur : Malakoff : Armand Colin, DL 2016, cop. 
15. Kouadio, L. S. (2015). Environnement psychosocial et maladie chronique : cas de la tuberculose multi résistante. Abidjan, Université Félix HouphouetBoigny.

16. Kouamé, A. (2017). « Réflexion sur les enjeux épistémologique et méthodologique de l'approche anthropologique sur la maladie chronique en contexte africain à travers l'exemple du VIH/Sida ", European Scientific Journal, Vol.13, No.29, pp $344-357$.

17. Kouamé, A. (2012). «Approche psycho qualitative du VIH/SIDA en contexte africain : cas du vécu de l'infection chez des patients du service des maladies infectieuses et tropicales (SMIT) d'Abidjan ». Recherches qualitatives - vol. 31(1), pp. 130-151.

18. Kouamé, A. (2010). "Vivre avec le VIH sous le regard des autres en milieu africain : l'expérience de séropositifs rencontrés à Abidjan », Revue Africaine d'Anthropologie, Nyansa-Pô, n' 10 -, PP 23- 40.

19. Kouamé, A. (2009). Conduites suicidaires chez les personnes vivant avec le VIH/Sida à Abidjan (Côte D'ivoire):Etude des facteurs de Risque associés, Thése de Doctorat Université de La Méditerranée (Aix Marseille 2), Université FHB de Cocody, 388 p.

20. Le Breton, D. (1985). Corps et sociétés : essai de sociologie et d'anthropologie du corps. Paris : Librairie des Méridiens.

21. Morse, J.M. (1991). Approaches to qualitative-quantitative methodological triangulation.Nursing Research, 40, 120-123

22. Morse, J.M. (1994). Critical issues in qualitative research methods. Thousand Oaks : Sage Publications.

23. Morse, J.M. \& Field, P.A. (1995). Qualitative research methods for health professionnals (second edition).SAGE publications.

24. Mayer, R. \& Deslauriers, JP. (2000). Quelques éléments d'analyse qualitative: l'analyse de contenu, l'analyse ancrée, l'induction analytique et le récit de vie. In Mayer R., Ouellet, F., Saint-Jacques, M.C.

25. Maier, B. \& Görgen, R. (1994). Assessment of the District Health System:Using qualitative methods. Institute of Tropical Hygiene/GTZ (Eds).London:MacMillan

26. Patton, M.Q. (1990). Qualitative evaluation and research methods (2nd éd.). Newbury Park, CA: SAGE.

27. Patton, M.Q. (2002). Qualitative research \& evaluation methods (3e éd.). Thousand Oaks, CA : Sage.

28. Savoie-Zajc, L. (1997). L'entrevue semi-dirigée. Dans B. Gauthier (Éd.), Recherche sociale. De la problématique à la collecte des données (3e éd., pp. 263-285). Québec : Presses de l'Université du Québec. 
29. Taylor, S. \& Bogdan, L. (1984). Introduction to qualitative research methods: the search for meaning.New York : Wiley.

30. Vidal, L. (1992). Sida et représentations de la maladie : Éléments de réflexion sur la séropositivité et sa prise en charge. Abidjan, Orstom, Cah. Sci. Hum. 28 (1) 7992 : 83-98.

31. Weber, M. (1965). "Essais sur la théorie de la science».

32. Yeo, T, J, M., Yessonguilana,J,M., Medar, K, A., Kone, A., \& Kone, D. (2012). PSY-CAUSE, Etude monographique du délai de la demande de soins chez les patients schizophrènes à l'hôpital psychiatrique de Bingerville (Côte d'Ivoire) n61, p. 25-30.

33. Yoro, B.M. (2012).Pluralisme thérapeutique et recours aux soins en milieu rural ivoirien : Approche méthodologique. Recherches qualitatives - Vol. 31(1), pp. 47-61. 